\title{
Chemoenzymatic synthesis and utilization of a SAM analog with an isomorphic nucleobase $\uparrow$
}

Cite this: Org. Biomol. Chem., 2016, 14,6189

Received 19th April 2016,

C. Vranken, ${ }^{a, b}$ A. Fin, ${ }^{b}$ P. Tufar, ${ }^{b}$ J. Hofkens, ${ }^{a}$ M. D. Burkart ${ }^{b}$ and Y. Tor ${ }^{\star b}$

Accepted 24th May 2016

DOI: $10.1039 / \mathrm{c} 60 \mathrm{~b} 00844$ e

www.rsc.org/obc

SalL, an enzyme that catalyzes the synthesis of SAM from L-methionine and $5^{\prime}$-chloro-5'-deoxyoadenosine, is shown to accept 5'-chloro-5'-deoxythienoadenosine as a substrate and facilitate the synthesis of a synthetic SAM analog with an unnatural nucleobase. This synthetic cofactor is demonstrated to replace SAM in the DNA methylation reaction with M.Taql.

Following ATP, $S$-adenosyl-t-methionine (SAM, also known as AdoMet) is the most ubiquitous and utilized adenosine-containing cofactor. ${ }^{1}$ SAM serves as the methyl donor in numerous methyltransferase (MTase)-catalyzed reactions, which are responsible for methylating both large biopolymers (e.g., DNA, RNA, proteins) and low molecular weight metabolites (e.g., catecholamines, polyketides). ${ }^{2,3}$ It has also been discovered to bind riboswitches, highlighting its regulatory roles in transcription and translation. ${ }^{4-6}$ As such, defects in SAM metabolism and its associated reactions are intimately linked to human health. ${ }^{7,8}$

Due to its universal role as the source of methyl groups, modified SAM analogs have been gaining utility in a variety of chemical and biological applications. ${ }^{2,9}$ Modified analogs, where the sulfonium center has been replaced by an aziridinyl ${ }^{10-15}$ or a (2-haloethyl)amino-group ( $N$-mustard), ${ }^{9,16-19}$ have been used to conjugate or crosslink biomolecules involved in SAMmediated transformations. ${ }^{9}$ In another class of SAM analogs, the methyl group has been replaced by a larger chemical entity that could directly be transferred to a substrate by methyl transferases, diverse SAM-dependent enzymes. ${ }^{20-23}$ Such modified SAM analogs can be used to further functionalize biomolecules through bioorthogonal conjugations, such as click reactions. Clickable groups have been used, for example, to biotinylate

\footnotetext{
${ }^{a}$ Department of Chemistry, KU Leuven, Celestijnenlaan 200F, 3001 Heverlee, Belgium ${ }^{b}$ Department of Chemistry and Biochemistry, University of California, San Diego, La Jolla, California 92093-0358, USA. E-mail: ytor@ucsd.edu

$\dagger$ Electronic supplementary information (ESI) available: Synthesis and characterization of new compounds, expression of SalL enzyme, enzymatic procedures and control experiments. See DOI: 10.1039/c6ob00844e
}

biomolecules or to conjugate diverse fluorophores to DNA for super-resolution optical DNA mapping. ${ }^{24,25}$

SAM analogs with isomorphic nucleobases have been minimally studied, ${ }^{26}$ and the tolerance of the enzymes involved in their biosynthesis and methylation reactions has not been broadly assessed. Such analogs can shed light on the fidelity of enzymes involved in metabolic processes and can potentially be used as mechanistic and biophysical probes. ${ }^{27}$ Furthermore, due to their distinct heterocyclic nucleus, conferring unique photophysical features, they can facilitate the fabrication of biophysical and inhibitor-discovery assays. ${ }^{28}$ Before advancing such applications, a viable enzymatic approach to
A

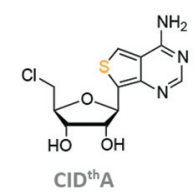

B

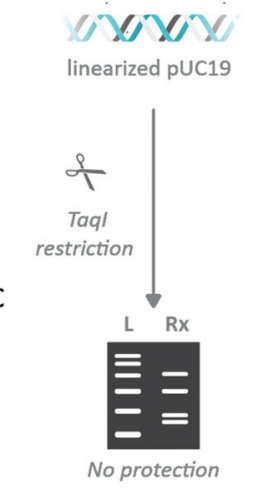

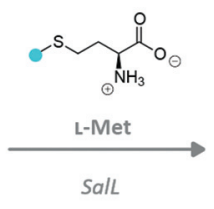
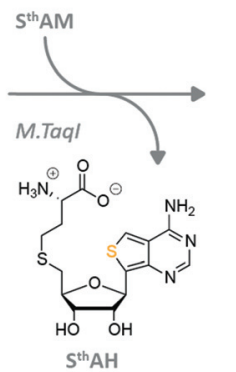

$\begin{array}{ll}- & \mathrm{CH}_{3} \\ \mathrm{~L} & \text { Ladder } \\ \text { Rx } & \text { Reaction }\end{array}$
Fig. 1 (A) $S^{\text {th }} A M$ is enzymatically prepared from $C D^{\text {th }} A$ and L-methionine using SalL. (B) Methylation of linearized pUC19 DNA with M.Taql and $S^{\text {th }} A M$ is followed by a strand cleavage step with Taql (a restriction enzyme). (C) Reactions are then analyzed by agarose gel electrophoresis. Methylation (protection) prevents any digestion and shows full length DNA. An unmethylated DNA shows full digestion after the exposure to a restriction enzyme. 
such molecules is needed, and their biochemical performance needs to be assessed.

SAM can be enzymatically synthesized by SAM synthetases using L-methionine and ATP. ${ }^{31,32}$ This reaction has been utilized to prepare a library of non-native S/Se-containing modified SAM analogs and to alkylate small molecules in situ. ${ }^{33}$ Alternatively, chemoenzymatic synthesis of several SAM analogs has been demonstrated using SalL, a chlorinase from Salinaspora tropica. ${ }^{34}$ This halogenase is known to naturally catalyze the breakdown of SAM to L-methionine and 5'-chloro5'-deoxyadenosine (CIDA), but the reaction can be reversed in vitro, at low chloride and high L-methionine concentrations. Using this enzyme, different SAM analogs were synthesized and utilized in situ to enzymatically alkylate a small molecule, a peptide and DNA. ${ }^{35,36}$

To explore whether or not such metabolic partners tolerate SAM analogs with unnatural heterocycles, which are not derived from adenosine, we have investigated the ability of SalL to synthesize $S^{\text {th }}$ AM, a SAM analog where adenosine has been replaced by a thieno[3,4- $d]$ pyrimidine-based adenosine surrogate (Fig. 1). This heterocycle is a member of a modified RNA alphabet we previously fabricated as a mechanistic and biophysical tool kit for exploring transformations of nucleosides, nucleotides and oligonucleotides. ${ }^{28,37-39}$ Here we demonstrate the successful SalL-mediated synthesis of $S^{\text {th }} A M$ from L-methionine and 5'-chloro-5'-deoxythienoadenosine $\left(\mathrm{ClD}^{\text {th }} \mathrm{A}\right)$. We then subsequently illustrate the applicability of $\mathrm{S}^{\text {th }} \mathrm{AM}$ as the methyl donor in methyl transferase-mediated DNA methylation. These results illustrate the broad tolerance of enzymes involved in the biosynthesis of SAM and its methyl transfer reactions to alterations of the adenosine moiety. ${ }^{26}$

The synthesis pathway to $\mathrm{S}^{\text {th }} \mathrm{AM}$ commences with the conversion of the nucleoside ${ }^{\text {th }} \mathrm{A}$ to its $5^{\prime}$-chloro- $5^{\prime}$-deoxy derivative $\mathrm{ClD}^{\text {th }} \mathrm{A}$ (Fig. 2A), following established procedures. ${ }^{29,30}$
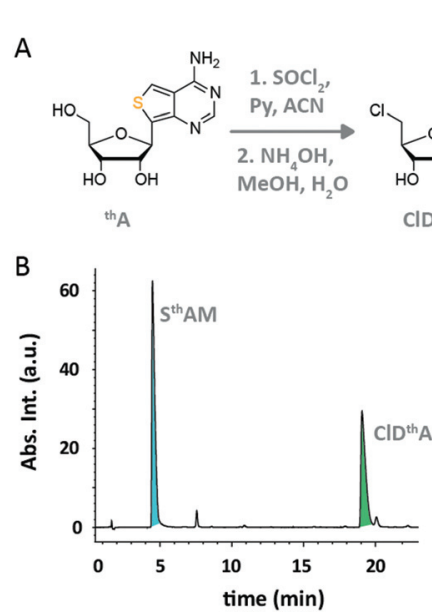
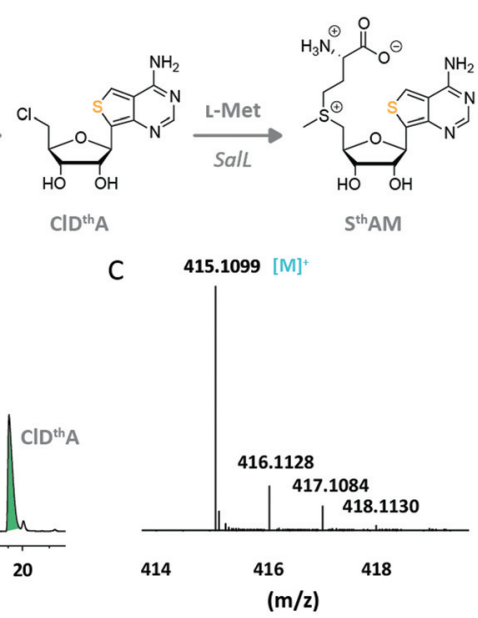

Fig. 2 (A) Synthesis of $S^{\text {th }} A M$. CID ${ }^{\text {th }} A$ is synthesized from the modified nucleoside ${ }^{\text {th }} A$ using an established chlorination method, ${ }^{29,30}$ which is then converted to $S^{\text {th }} A M$ using L-methionine and SalL. (B) HPLC trace of a typical enzymatic $S^{\text {th }} A M$ synthesis reaction, containing $0.2 \mathrm{mM} \mathrm{CID}^{\text {th }} \mathrm{A}$, $15 \mathrm{mM} \mathrm{L}-$ Met and $9 \mu \mathrm{M}$ SalL for 2 hours at $37^{\circ} \mathrm{C}$. (C) HR-MS trace of $S^{\text {th }} A M$, purified using HPLC.
Incubating this halogenated non-native nucleoside with SalL and a large excess of L-methionine $(\times 75)$ at $37^{\circ} \mathrm{C}$ generates $S^{\text {th }} \mathrm{AM}$, the modified analog. The formation of the desired product during the enzymatic reaction was monitored by HPLC and confirmed by HR-MS (Fig. 2B, C, S3 and S7 + ). The production of $\mathrm{S}^{\text {th }} \mathrm{AM}$ could be scaled up and optimized by increasing reaction volumes and using higher concentrations of the enzyme. The best conversion we observed, as reflected by the highest ratio of the product $\left(\mathrm{S}^{\text {th }} \mathrm{AM}\right)$ to the starting material $\left(\mathrm{ClD}^{\mathrm{th}} \mathrm{A}\right)$, was 1.38 , reflecting the consumption of about $60 \%$ of the chlorinated nucleoside (Fig. S4 $\dagger$ ). Control experiments show no formation of $S^{\text {th }} A M$ in the absence of the enzyme excluding any direct substitution reaction between L-methionine and $\mathrm{ClD}^{\text {th }} \mathrm{A}$ (Fig. S6 $†$ ).

To study its capability as a methylating agent, $\mathrm{S}^{\text {th }} \mathrm{AM}$ was used in a DNA methylation-restriction assay with a common DNA substrate (a pUC19 plasmid linearized by SacI) and an M.TaqI methyl transferase (MTase). This enzyme naturally catalyzes the methylation of the exocyclic amino group of adenine within the 5'-TCGA-3' sequence in double-stranded DNA. The methylation reaction can then be followed by incubation with the endonuclease TaqI. This enzyme has the same recognition sequence as M.TaqI, but is unable to cleave the methylated DNA sequences. Finally, the reactions are analyzed by agarose gel electrophoresis using ethidium bromide staining (Fig. 1). A control reaction with SAM, the natural cofactor, can be executed in parallel to compare the two methyl donors.

As seen in Fig. 3, after an hour long reaction, a nearly complete protection was observed with $S^{\text {th }}$ AM (Fig. 3, lane 1), compared to a complete reaction with SAM, the native cofactor

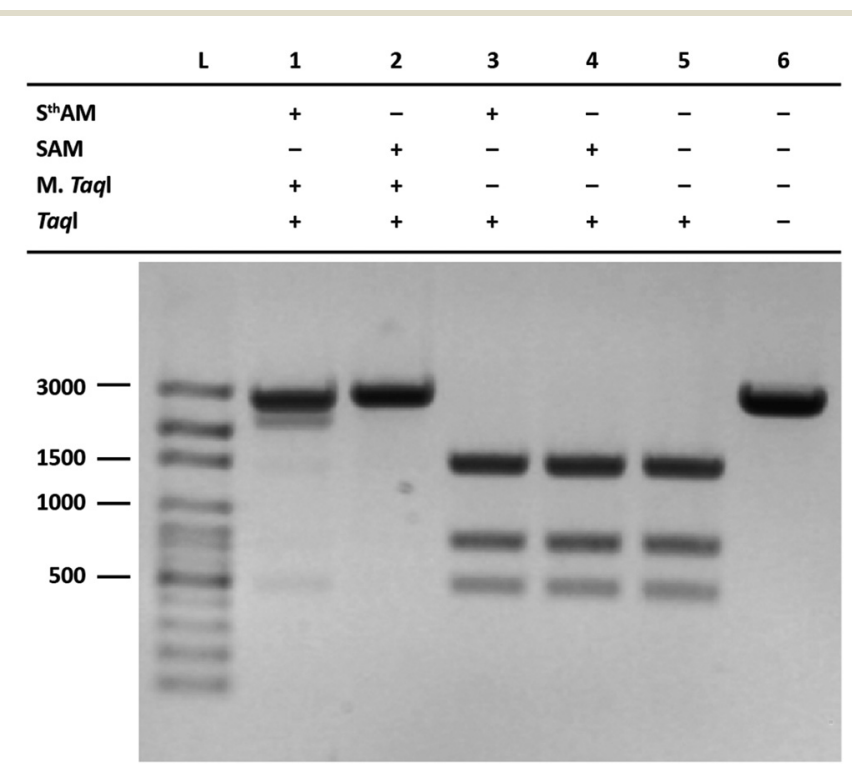

Fig. 3 Methylation of linearized pUC19 DNA by M.Taql MTase. A DNA marker (100-3000 bp) was used as a reference (lane L). Lanes 1 and 2 show the result after the reaction using $S^{\text {th }} A M$ and SAM respectively. Negative controls in the absence of M.Taql for the reaction with $S^{\text {th }} A M$ (lane 3), SAM (lane 4) or no cofactor (lane 5) are shown. Lane 6 shows a control reaction in the absence of any cofactor or enzyme. 
(lane 2). Control reactions (lanes 3-6) confirm the need for all components for a successful DNA protection.

To semi-quantitatively compare the reaction rates of M.TaqI with SAM and $S^{\text {th }}$ AM, the DNA methylation reaction was followed over a 2 hour time course (Fig. 4). Five time points were taken for each cofactor after 10, 30, 45, 105 and $120 \mathrm{~min}$ exposure to the MTase, followed by incubation with TaqI. While the reaction with SAM was virtually quantitative after $10 \mathrm{~min}$ (lanes 6-10), the reaction with $\mathrm{S}^{\text {th }} \mathrm{AM}$ was slower but essentially reached completion after $2 \mathrm{~h}$ (lanes 1-5).

To test whether $\mathrm{S}^{\text {th }} \mathrm{AM}$ can be formed in situ and used directly in a methylation reaction, SalL and M.TaqI were combined in a one-pot reaction (Fig. 5). This combined synthesis/ methylation reaction and the subsequent cleavage were performed for both ClDA (lane 1) and $\operatorname{ClD}^{\text {th }} \mathrm{A}$ (lane 2). Positive controls in which SAM was added in the absence of SalL (lanes 3 and 4) were also performed. Negative controls in which SalL (lanes 5 and 6), L-Met (lanes 7 and 8), ClDA/ClD ${ }^{\text {th }} \mathrm{A}$ (lane 9) or M.TaqI (lanes 10 and 11) was omitted and one control with only DNA (lanes 12 and 13) were performed under the same conditions. The reaction with ClDA (lane 1) showed full protection. The one where $\mathrm{ClD}^{\text {th }} \mathrm{A}$ was used (lane 2) shows partial protection and the negative controls show no protection as expected, with the exception of lane 9, where one extra band with a higher molecular weight compared to the largest possible fragment is present. This extra band, indicating partial DNA protection, could result from small amounts of in situ generated SAM. The latter is formed from traces of CIDA bound to SalL, which has been reported to co-purify with A and ClDA when expressed in chloride-containing media. ${ }^{34}$ As exogenous $\mathrm{ClDA} / \mathrm{ClD}^{\text {th }} \mathrm{A}$ is absent in lane 9, traces of SAM facilitate a small, yet observable, protection and therefore

\begin{tabular}{ccccc}
+ & - & - & - \\
- & + & - & - \\
+ & + & - & - \\
+ & + & + & - \\
\hline
\end{tabular}

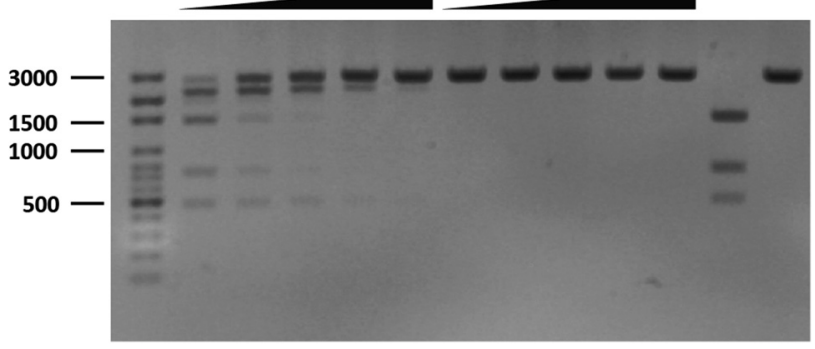

Fig. 4 Time course methylation-restriction assay of linearized pUC19 DNA by M.Taql methyltransferase. A 100 bp ladder DNA marker (100-3000 bp) was used as a reference (lane L). During the methylation reaction, five time points were taken for both $S^{\text {th }} A M$ (first group of time points) and SAM (second group of time points). After treatment with Taql, the reactions were analyzed on a $1 \%$ agarose gel. The DNA lane shows the control in the absence of M.Taql and the cofactor, in the presence and absence of Taql.

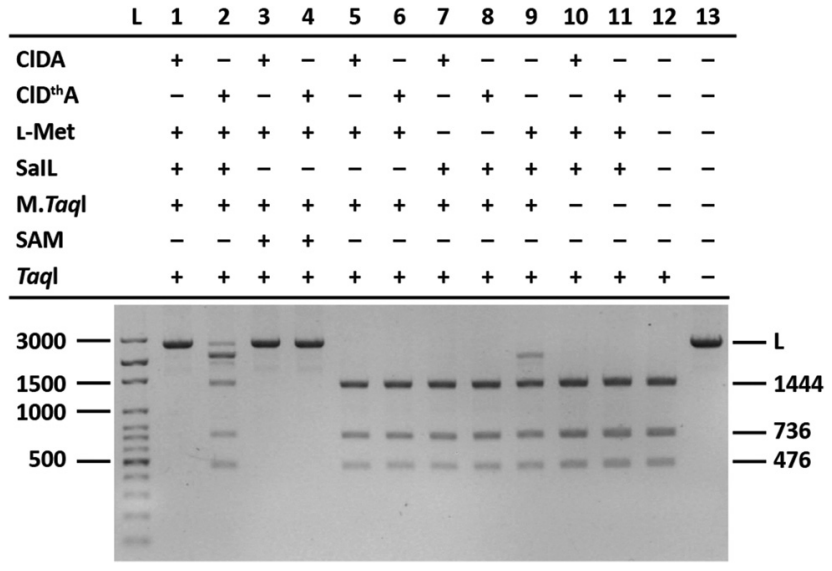

Fig. 5 Combination synthesis-methylation-restriction assay using linearized pUC19 DNA, SalL and M.Taql. Lane L is a 100 bp ladder DNA marker (100-3000 bp). Lanes 1 and 2 are the combination reactions with respectively CIDA and $C D^{\text {th }} A$. Lanes 3 and 4 are positive controls in the presence of SAM, but the absence of SalL. Negative controls with respectively CIDA and $\mathrm{CID}^{\text {th } A}$ were performed in the absence of SalL (lanes 5 and 6), of L-Met (lanes 7 and 8) and of M.Taql (lanes 10 and 11). Controls without $\mathrm{CID}^{\text {th }} \mathrm{A}$ (lane 9) and only DNA (lane 13) are shown.

leads to a partly "false positive" result. Nevertheless, as the full length DNA is not present in this control lane, but is present in lane 2, the protection seen in the latter must be due to the formation of $\mathrm{S}^{\text {th }} \mathrm{AM}$.

Although $S^{\text {th }}$ AM appears to be somewhat inferior to SAM in its enzymatic synthesis and methyl transfer reactions, it is rather remarkable that the enzymes involved in its synthesis and utilization show such tolerance for nucleobase modifications. In particular, while the halogenated adenosine has been crystallographically shown to be buried within the SalL active site ${ }^{34}$ with the Asn188's amide side chain forming two $\mathrm{H}$ bonds to the Hoogsteen face of adenine, the enzyme tolerates the replacement of the imidazole unit in adenine with a thiophene. In contrast, M.TaqI does not appear to form specific contacts with the adenine's imidazole moiety, ${ }^{40}$ which may explain its higher tolerance and ability to effectively utilize $S^{\text {th }} A M$ in DNA methylation reactions (Fig. S8 $\dagger$ ).

\section{Conclusions}

A chemoenzymatic synthesis of $S^{\text {th }} A M$, a new functional SAM analog, has been accomplished by combining L-methionine and $\mathrm{ClD}^{\text {th }} \mathrm{A}$ in a reaction catalyzed by SalL, an enzyme known to perform a similar reaction with ClDA. The new synthetic cofactor could be used for DNA methylation reactions in a methylation-restriction assay using a pUC19 vector and M.TaqI MTase. These results highlight the isomorphicity of ${ }^{\text {th }} \mathrm{A}$, a new adenosine isoster, and the tolerance level of enzymes involved in the biosynthesis and utilization of SAM. The availability of functional analogs with altered nucleobases can also facilitate future biophysical analyses and mechanistic studies of biochemical processes involving this abundant cofactor. Our 
observations suggest that ${ }^{\text {th }} \mathrm{A}$, a highly isomorphic adenosine surrogate, may be able to substitute the native nucleoside in other cofactors and second messengers.

\section{Acknowledgements}

Support to J. H. from the European Research Council under the European Union's Seventh Framework Program [FP7/ 2007-2013]/ERC Grant Agreement [291593 FLUOROCODE] and the Flemish government in the form of long-term structural funding 'Methusalem' [METH/08/04 CASAS] was gratefully acknowledged. C.V. thanks the KU Leuven for the JuMo scholarship. We thank the US National Institutes of Health for generous support (GM 069773 to Y. T.), and UCSD's Chemistry \& Biochemistry MS Facility.

\section{Notes and references}

1 H. L. Schubert, R. M. Blumenthal and X. Cheng, Trends Biochem. Sci., 2003, 28, 329-335.

2 J. Zhang and Y. G. Zheng, ACS Chem. Biol., 2016, 11, 583597.

3 A. W. Struck, M. L. Thompson, L. S. Wong and J. Micklefield, ChemBioChem, 2012, 13, 2642-2655.

4 W. C. Winkler and R. R. Breaker, Annu. Rev. Microbiol., 2005, 59, 487-517.

5 B. A. McDaniel, F. J. Grundy and T. M. Henkin, Mol. Microbiol., 2005, 57, 1008-1021.

6 J. J. Trausch, Z. Xu, A. L. Edwards, F. E. Reyes, P. E. Ross, R. Knight and R. T. Batey, Proc. Natl. Acad. Sci. U. S. A., 2014, 111, 6624-6629.

7 Q. M. Anstee and C. P. Day, J. Hepatol., 2012, 57, 10971109.

8 D. Mischoulon and M. Fava, Am. J. Clin. Nutr., 2002, 76, 1158S-1161S.

9 H. Hu, K. Qian, M.-C. Ho and Y. G. Zheng, Expert Opin. Investig. Drugs, 2016, 25, 335-358.

10 L. R. Comstock and S. R. Rajski, J. Am. Chem. Soc., 2005, 127, 14136-14137.

11 L. R. Comstock and S. R. Rajski, Nucleic Acids Res., 2005, 33, 1644-1652.

12 M. Pignot, C. Siethoff, M. Linscheid and E. Weinhold, Angew. Chem., Int. Ed., 1998, 37, 2888-2891.

13 G. Pljevaljcic, M. Pignot and E. Weinhold, J. Am. Chem. Soc., 2003, 125, 3486-3492.

14 G. Pljevaljcic, F. Schmidt, A. Peschlow and E. Weinhold, Methods Mol. Biol., 2004, 283, 145-161.

15 G. Pljevaljcic, F. Schmidt and E. Weinhold, ChemBioChem, 2004, 5, 265-269.

16 R. L. Weller and S. R. Rajski, Org. Lett., 2005, 7, 2141-2144.

17 R. L. Weller and S. R. Rajski, ChemBioChem, 2006, 7, 243-245.
18 S. J. Hymbaugh Bergman and L. R. Comstock, Bioorg. Med. Chem., 2015, 23, 5050-5055.

19 Y. Du, C. E. Hendrick, K. S. Frye and L. R. Comstock, ChemBioChem, 2012, 13, 2225-2233.

20 C. Dalhoff, G. Lukinavicius, S. Klimasauskas and E. Weinhold, Nat. Protoc., 2006, 1, 1879-1886.

21 S. Klimasauskas and E. Weinhold, Trends Biotechnol., 2007, 25, 99-104.

22 L. W. Parks, J. Biol. Chem., 1958, 232, 169-176.

23 F. Schlenk and J. L. Dainko, Biochim. Biophys. Acta, 1975, 385, 312-323.

24 C. Vranken, J. Deen, L. Dirix, T. Stakenborg, W. Dehaen, V. Leen, J. Hofkens and R. K. Neely, Nucleic Acids Res., 2014, 42, e50.

25 R. K. Neely, P. Dedecker, J.-i. Hotta, G. Urbanaviciute, S. Klimasauskas and J. Hofkens, Chem. Sci., 2010, 1, 453460.

26 O. M. Ottink, F. H. T. Nelissen, Y. Derks, S. S. Wijmenga and H. A. Heus, Anal. Biochem., 2010, 396, 280-283.

27 N. J. Leonard, CRC Crit. Rev. Biochem., 1984, 15, 125-199.

28 R. W. Sinkeldam, L. S. McCoy, D. Shin and Y. Tor, Angew. Chem. Int. Ed., 2013, 52, 14026-14030.

29 D. P. C. McGee and J. C. Martin, Can. J. Chem., 1986, 64, 1885-1889.

30 A. Micoli, A. Turco, E. Araujo-Palomo, A. Encinas, M. Quintana and M. Prato, Chem. - Eur. J., 2014, 20, 53975402.

31 G. L. Cantoni, J. Am. Chem. Soc., 1952, 74, 2942-2943.

32 A. Gross, S. Geresh and G. M. Whitesides, Appl. Biochem. Biotechnol., 1983, 8, 415-422.

33 S. Singh, J. Zhang, T. D. Huber, M. Sunkara, K. Hurley, R. D. Goff, G. Wang, W. Zhang, C. Liu, J. Rohr, S. G. Van Lanen, A. J. Morris and J. S. Thorson, Angew. Chem., Int. Ed., 2014, 53, 3965-3969.

34 A. S. Eustaquio, F. Pojer, J. P. Noel and B. S. Moore, Nat. Chem. Biol., 2008, 4, 69-74.

35 J. M. Lipson, M. Thomsen, B. S. Moore, R. P. Clausen, J. J. La Clair and M. D. Burkart, ChemBioChem, 2013, 14, 950-953.

36 M. Thomsen, S. B. Vogensen, J. Buchardt, M. D. Burkart and R. P. Clausen, Org. Biomol. Chem., 2013, 11, 76067610.

37 D. Shin, R. W. Sinkeldam and Y. Tor, J. Am. Chem. Soc., 2011, 133, 14912-14915.

38 R. A. Mizrahi, D. Shin, R. W. Sinkeldam, K. J. Phelps, A. Fin, D. J. Tantillo, Y. Tor and P. A. Beal, Angew. Chem., Int. Ed., 2015, 54, 8713-8716.

39 D. Shin, P. Lonn, S. F. Dowdy and Y. Tor, Chem. Commun., 2015, 51, 1662-1665.

40 J. Labahn, J. Granzin, G. Schluckebier, D. P. Robinson, W. E. Jack, I. Schildkraut and W. Saenger, Proc. Natl. Acad. Sci. U. S. A., 1994, 91, 10957-10961. 